\title{
SACARIFICAÇÃO DE PAPEL DE ESCRITÓRIO RESIDUAL PARA OBTENÇÃO DE ETANOL DE SEGUNDA GERAÇÃO
}

\author{
P. P. K. A. LADEIRA ${ }^{1}$, G. das G. PEREIRA ${ }^{1}$ e F. P. CASCIATORI ${ }^{2}$ \\ ${ }^{1}$ Universidade Federal de São Carlos, Centro de Ciências da Natureza \\ ${ }^{2}$ Universidade Federal de São Carlos, Departamento de Engenharia Química \\ E-mail para contato: pedrokropfengambiental@gmail.com
}

\begin{abstract}
RESUMO - O desenvolvimento tecnológico de uma matriz energética limpa e renovável é estratégico para o Brasil, de modo que a busca por viabilizar o aproveitamento de resíduos celulósicos na produção do chamado etanol de segunda geração por via bioquímica tem sido investigada em diferentes vertentes. No presente trabalho, propôs-se empregar enzimas produzidas por cultivo sólido na sacarificação de papel de escritório residual, possível fonte de açúcares fermentáveis a partir de degradação da celulose. Foram testados o papel in natura e submetido a um pré-tratamento combinado (físico-químico) sobre a eficiência de sacarificação, bem como diferentes dosagens de enzima (4 e $8 \mathrm{~mL}$ ) e tempos de hidrólise $(8$ e $24 \mathrm{~h})$. O papel com pré-tratamento foi o que mais liberou ART, atingindo aproximadamente $27 \mathrm{mg}$ ART liberados por grama de papel, porém na média os resultados não diferiram significativamente, indicando ser mais interessante empregar o papel in natura, poupando o uso de energia e de reagentes químicos. Já com relação ao tempo de hidrólise, o mesmo não influenciou significativamente na liberação de ART e o volume de extrato enzimático só apresentou influência significativa na liberação de ART a partir do papel submetido ao pré-tratamento. As melhores condições foram definidas como: papel in natura, volume de extrato $4 \mathrm{~mL}$ e tempo de hidrólise 8 horas, tendo em vista economia de energia, enzima e reagentes.
\end{abstract}

\section{INTRODUÇÃO}

O fornecimento de energia está embasado tanto na estruturação quanto na dinâmica operacional da sociedade, abrangendo desde o bem-estar individual até o desempenho industrial e de prestações de serviços. O petróleo, fundamental nesse fornecimento, é responsável por um terço da energia primária consumida no planeta (ODAC, 2007). Contudo, devido a tratar-se de uma matriz não-renovável e ainda a sua respectiva combustão, recorrente há 60 anos, gerar o acúmulo de gases como o $\mathrm{CO}_{2}$, que intensificam o efeito estufa e alterações climáticas, tem sido fomentada a busca por fontes renováveis de energia (Pereira Jr. et al., 2008).

\subsection{Etanol de Primeira e Segunda Geração}

O etanol convencional ou etanol de primeira geração (E1G) origina-se a partir da fermentação do caldo da cana-de-açúcar, rico em sacarose. As estratégias para aumento da 
produção de etanol incluem aumento da área de cana plantada, aumento de produtividade das usinas ou emprego de biomassa residual como matéria-prima. Essa última alternativa se traduz como a tecnologia de produção do denominado etanol de segunda geração (E2G), que tem como matéria-prima açúcares provenientes da quebra das cadeias de celulose de biomassa residual, por exemplo, e tem sido incentivada pelos governos federal e estadual paulista desde 2010, quando foi criado o Centro Paulista de Pesquisa em Bioenergia, visando a impulsionar estudos na área.

Neste contexto da possibilidade do emprego de diferentes tipos de biomassa residual celulósica para obtenção de E2G, pode-se destacar o papel, que pode ser considerado como importante matéria-prima para a produção de bioetanol, uma vez que congrega características de ser um substrato de baixo valor agregado, abundante e de fácil disponibilidade. Sua composição é basicamente celulósica, de forma que a hidrólise da cadeia pode levar à obtenção de açúcares fermentescíveis (Maiti et al., 2011).

\subsection{Vias de Obtenção de Etanol de Segunda Geração}

O processo envolvendo a conversão de biomassa celulósica a E2G consiste basicamente em quatro etapas: pré-tratamento, hidrólise, fermentação e destilação. O prétratamento é essencial para tornar as fibras de celulose mais disponíveis para a hidrólise, que por sua vez consiste na conversão dos carboidratos das cadeias de celulose a açúcares fermentescíveis, podendo ser feita por rota química ou enzimática. Finalmente, os açúcares fermentescíveis liberados podem ser fermentados e o caldo fermentado pode seguir para a destilação, como no processo convencional de produção do E1G. Os pré-tratamentos físicoquímicos consistem de reações químicas realizadas a altas temperaturas e pressões. Atualmente, as principais pesquisas são voltadas para tecnologias desse tipo de prétratamento, que tem como exemplos: explosão à vapor, explosão com $\mathrm{CO} 2$ e tratamento com água quente.

A etapa seguinte ao pré-tratamento, de hidrólise da celulose a açúcares fermentescíveis ou sacarificação, pode ocorrer por duas vias: enzimática ou química. A via química, embora eficiente e rápida, gera resíduos tóxicos que devem ser tratados, aumentando o número de operações, consumindo energia e tornando o processo pouco competitivo. A hidrólise enzimática apresenta diversas vantagens sobre a química, dentre as quais se devem destacar o menor gasto energético e a alta especificidade pelo substrato, evitando a produção de compostos indesejáveis. As principais enzimas envolvidas neste processo são as celulases, nome genérico usado para designar uma classe de enzimas que atuam conjuntamente na quebra da celulose a glicose, sendo constituídas por endoglucanases (quebra a celulose na região amorfa e libera os celo-oligossacarídeos), exoglucanases (quebra a celulose e os celooligossacarídeos maiores e libera a celobiose) e $\beta$-glucosidase (cliva os dímeros celobiose e finalmente libera as moléculas de glicose) (Pinto, 2010). No entanto, a via enzimática é mais lenta e tem como uma das principais barreiras o custo das enzimas requeridas. Uma alternativa eficiente que vem sendo estudada para a produção dessas enzimas a serem aplicadas na hidrólise da biomassa celulósica visando a obtenção de E2G a custo que viabilize seu uso (Mishima et al., 2006) é o cultivo em estado sólido (CES).

O cultivo em estado sólido (CES) pode ser definido como o crescimento de microrganismos sobre partículas sólidas úmidas, em situações nas quais o espaço entre as 
partículas contém uma fase gasosa contínua e a água está ou impregnada nas partículas ou forma um fino filme de água sobre elas. Entretanto, o conteúdo de água da matriz porosa deve ser suficientemente alto para assegurar adequadas condições de crescimento ao metabolismo microbiano (Mitchell et al., 2006). Devido às atividades de água mais baixas encontradas nos sistemas de CES, os microrganismos que mais se adaptam a esse tipo de cultivo são os fungos filamentosos, para os quais o CES reproduz o habitat natural, o que faz com que estes excretem naturalmente grandes quantidades de enzimas para o meio extracelular. Dentre estes fungos filamentosos, os termofílicos apresentam a vantagem adicional de produzir enzimas mais termoestáveis, uma característica bastante interessante do ponto de vista industrial da produção de $\mathrm{E} 2 \mathrm{G}$, sobretudo quando pré-tratamentos que envolvem o uso de calor são empregados na etapa anterior à hidrólise enzimática (Da Silva et al., 2005). Como as enzimas produzidas por fungos termofílicos atuam bem em temperaturas mais elevadas, é possível gastar uma quantidade de energia menor para resfriamento do material entre as operações de pré-tratamento a quente e de sacarificação, aumentando a viabilidade do processo.

Em trabalhos anteriores do grupo de pesquisa em Bioenergia do Ibilce/UNESP, foi selecionado o fungo termofílico Myceliophtora thermophila I-1D3b como bom produtor de celulases. Já foram realizados alguns estudos de aplicação dos extratos enzimáticos produzidos por este fungo em CES na sacarificação de bagaço de cana in natura e pré-tratado e resultados promissores foram reportados (Pinto, 2010; Travaini, 2011; Casciatori, 2015), denotando a viabilidade de se testar sua aplicação em outros substratos celulósicos passíveis de serem destinados à produção de $\mathrm{E} 2 \mathrm{G}$.

Neste projeto, propôs-se empregar enzimas produzidas por cultivo sólido na hidrólise de papel residual, visando avaliar a liberação de açúcares fermentescíveis a partir da celulose.

\section{METODOLOGIA}

Foram comparados papel sem tratamento (in natura) e papel submetido a tratamento combinado. Também foram testados dois tempos de hidrólise ( 8 e $24 \mathrm{~h})$ e ainda se variou o volume de extrato enzimático $(4 \mathrm{e} 8 \mathrm{~mL})$. O tratamento combinado consistiu em aquecer $10 \mathrm{~g}$ de papel pré-umidificado com $10 \mathrm{~mL}$ de solução $\mathrm{HCl} 0,1 \mathrm{M}$ em autoclave $\left(121{ }^{\circ} \mathrm{C}\right.$ por 20 minutos); a amostra in natura foi apenas umidificada. Antes da hidrólise, os papéis foram secos em estufa até peso constante.

Para os testes de hidrólise, $0,5 \mathrm{~g}$ de papel foram pesados em seis frascos de Erlenmeyer e acrescidos de $4 \mathrm{~mL}$ (três frascos) e $8 \mathrm{~mL}$ (três frascos) de extrato enzimático produzido pelo fungo Myceliophthora thermophila I-1D3b em cultivo sólido, sendo o volume final completado para $10 \mathrm{~mL}$ com solução tampão acetato em $\mathrm{pH}=5$. A hidrólise ocorreu em incubadora com convecção forçada de ar e agitação orbital a $120 \mathrm{rpm}$. A temperatura foi mantida em $50{ }^{\circ} \mathrm{C}$, haja vista as enzimas serem termofílicas.

Para determinação do teor de açúcares redutores totais (ART) no hidrolisado, adicionaram-se, em tubos de ensaio, $0,5 \mathrm{~mL}$ de hidrolisado e $0,5 \mathrm{~mL}$ de reagente contendo ácido-3,5-di-nitro-salicílico. Os tubos foram levados à fervura por $10 \mathrm{~min}$, após o que foram resfriados em gelo e adicionados de $4 \mathrm{~mL}$ de água, procedendo-se à leitura da absorbância em espectrofotômetro a $540 \mathrm{~nm}$. Com base na curva padrão de glicose e no volume do meio 
reacional por grama de papel, os resultados foram expressos em mg de ART por grama de papel. Os ensaios foram feitos em triplicata.

\section{RESULTADOS E DISCUSSÃO}

Os resultados são apresentados na Tabela 1, onde se observa que foi obtida maior liberação de ART a partir de papel pré-tratado (com aproximadamente $27 \mathrm{mg}$ de ART por grama de papel), contudo nas análises estatísticas de comparação das médias realizadas com auxílio do software livre Chemoface v1.5 (UFLA, Lavras-MG, Brasil), foi possível notar que o mesmo não teve efeito significativo sobre a liberação de ART a partir do papel in natura e pré-tratado, conforme mostra a Figura 1. Ainda, quanto a interação entre as variáveis tempo de hidrólise e volume de extrato enzimático aplicado, a mesma só foi significativa somente para o papel pré-tratado, para o qual também se observou efeito de interação entre o volume de extrato enzimático e o tempo de hidrólise.

Tabela 1 - Liberação de ART a partir da hidrólise enzimática de papel residual

\begin{tabular}{|c|c|c|c|}
\hline Amostras & $\begin{array}{c}\text { Tempo de hidrólise } \\
\text { (h) }\end{array}$ & $\begin{array}{c}\text { Volume de } \\
\text { extrato } \\
\text { enzimático }(\mathrm{mL})\end{array}$ & $\begin{array}{c}\text { ART (mg / g papel) } \\
\text { [Média } \pm \text { desvio } \\
\text { padrão] }\end{array}$ \\
\hline \multirow{4}{*}{ In natura } & \multirow{2}{*}{8} & 4 & $18,1 \pm 0,2$ \\
\hline & & 8 & $20,7 \pm 1,5$ \\
\hline & \multirow{2}{*}{24} & 4 & $12,4 \pm 0,5$ \\
\hline & & 8 & $11,2 \pm 2,1$ \\
\hline \multirow{4}{*}{ Pré-tratado } & \multirow{2}{*}{8} & 4 & $18,8 \pm 0,3$ \\
\hline & & 8 & $27,1 \pm 7,5$ \\
\hline & \multirow{2}{*}{24} & 4 & $21,8 \pm 3,2$ \\
\hline & & 8 & $7,3 \pm 0,5$ \\
\hline
\end{tabular}

Figura 1 - Gráfico box-plot das médias de ART liberados por grama de papel in natura e pré-tratado

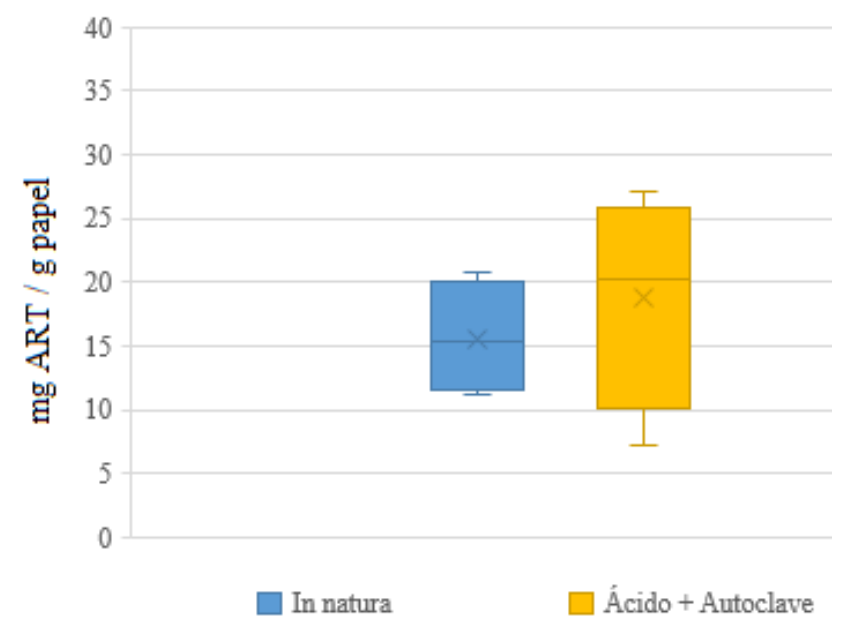


No caso do papel pré-tratado, observou-se que, para o tempo de 8 horas de hidrólise, a liberação de ART foi maior quando se empregou maior volume de extrato enzimático. Por outro lado, para 24 horas de hidrólise, a liberação de ART foi menor quando se aumentou o volume de extrato aplicado. Considerando que houve evaporação do excipiente líquido, tais resultados podem estar associados ao efeito da presença de íons hidrogênio livres $\left(\mathrm{H}^{+}\right)$no meio reacional no caso da aplicação dos pré-tratamento ácido combinado com autoclave e ao volume de solução tamponante empregado. Para o maior volume de extrato enzimático, menor volume de solução tampão foi utilizado. Considerando a maior evaporação nas 24 horas de hidrólise, a concentração de íons $\mathrm{H}^{+}$em solução pode ter atingido um nível tal que o menor volume de tampão não foi suficiente para manter o $\mathrm{pH}$ na faixa ótima de atuação da enzima. Por outro lado, tendo havido menor evaporação com 8 horas de hidrólise, possivelmente mesmo o menor volume de tampão empregado no caso do uso de maior volume de extrato enzimático foi suficiente para manter o $\mathrm{pH}$ na faixa desejada, favorecendo a atuação da enzima em seu estado de ionização que proporcionasse maior atividade catalítica (Borzani, 2001).

Como rendimento teórico, a máxima liberação de ART permitiria obter cerca de 13,5 L de etanol por tonelada de papel. Casciatori (2015), que realizou a hidrólise enzimática de bagaço de cana com o extrato produzido a partir do mesmo fungo empregado neste resumo e utilizando as mesmas considerações, obteve a estimativa da produção de $36,7 \mathrm{~L}$ de etanol por tonelada de bagaço de cana in natura. Assim, comparando-se ambos os substratos, tem-se que a produção de etanol a partir do papel corresponde a $37 \%$ da produção esperada a partir de bagaço de cana. Penariol et al. (2015), que também utilizou bagaço de cana in natura e realizou a hidrólise enzimática com o extrato produzido a partir do mesmo fungo $M$. thermophila, obteve a liberação de ART de aproximadamente $70 \mathrm{mg} / \mathrm{g}$ de bagaço. Novamente, quando comparados ambos os substratos, é possível notar que a liberação de ART por grama de papel in natura (cerca de 20,7 mg de ART por grama de papel) representa cerca de $30 \%$ do obtido a partir do bagaço de cana. No entanto, considerando que tanto o bagaço quanto o papel são resíduos, toda e qualquer forma de aproveitamento destes para a geração de bioenergia deve ser considerada válida, sobretudo se for realizada de forma integrada a biorrefinarias e se empregar o mínimo possível de recursos químicos e energéticos na sua conversão.

\section{AGRADECIMENTOS}

Os autores agradecem o apoio financeiro da Fundação de Amparo à Pesquisa do Estado de São Paulo (FAPESP) (Processo \#2016/10750-5).

\section{REFERÊNCIAS}

BORZANI, W. Cinética de reações enzimáticas. In: BORZANI, W.; SCHMIDELL, W.; LIMA, U. A.; AQUARONE, E. (Eds.). Biotecnologia Industrial. Vol. 1 - Fundamentos. São Paulo: Editora Edgar Blücher Ltda, 2001. Capítulo 7, p. 197 - 216.

CASCIATORI, P. A. Produção de enzimas celulolíticas pelos fungos Trichoderma reesei e Myceliophthora thermophila e aplicação na sacarificação do bagaço de cana-de-açucar. 
2015. 98 f. Dissertação (Mestrado). Universidade Estadual Paulista Júlio de Mesquita Filho, Instituto de Biociências, Letras e Ciências Exatas, São José do Rio Preto, 2015.

DA SILVA, R.; LAGO, E. S.; MERHEB, C. W.; MACCHIONE, M. M.; PARK, Y. K. Production of xylanase and CMCase on solid state fermentation in different residues by Thermoascus aurantiacus Miehe. Brazilian Journal of Microbiology, v. 36, p. 235 $241,2005$.

MAITI, B.; RATHORE, A.; SRIVASTAVA, S.; SHEKHAWAT, M.; SRIVASTAVA, P. Optimization of process parameters for ethanol production from sugar cane molasses by Zymomonas mobilis using response surface methodology and genetic algorithm. Applied Microbiology and Biotechnology, v. 90, n. 1, p. 385-395, 2011.

MISHIMA, D.; TATEDA, M.; IKE, M.; FUJITA, M. Comparative study on chemical pretreatments to accelerate enzymatic hydrolysis of aquatic macrophyte biomass used in water purification processes. Bioresource Technology, v. 97, p. 2166-2172, 2006.

MITCHELL, D. A.; KRIEGER, N.; BEROVIC, M. Solid-state fermentation bioreactors: fundamentals, design and operation. Springer-Verlag: Berlin, 2006.

ODAC. The Oil Depletion Analysis Centre. 2007. Diponível em: www.odac-info.org. Acesso em: Maio de 2016.

PENARIOL, B. R.; THOMÉO, J. C.; CASCIATORI, F. P. Hidrólise Enzimática de Bagaço de Cana in Natura empregando Celulases Produzidas por Fungos em Cultivo Sólido. São José do Rio Preto. 2015.

PEREIRA Jr., N.; COUTO, M. A. P. G.; SANTA ANNA, L. M. M. Biomass of lignocellulosic composition for fuel ethanol production and the context of biorefinery. In: Series on Biotechnology. Rio de Janeiro: Editora Amiga Digital UFRJ, v. 2, 45 p., 2008.

PINTO, T. O. P. Produção de enzimas celulolíticas pelos fungos Thermoascus aurantiacus CBMAI 756, Thermomyces lanuginosus, Trichoderma reesei QM9414 e Penicillium viridicatum RFC3 e aplicação na sacarificação do bagaço de cana de açúcar com diferentes pré-tratamentos. 2010. 91 f. Dissertação (Mestrado). Universidade Estadual Paulista Júlio de Mesquita Filho, Instituto de Biociências, Letras e Ciências Exatas, São José do Rio Preto, 2010.

TRAVAINI, R. Utilização de processos oxidativos avançados baseados em ozônio como prétratamento para bagaço de cana-de-açúcar, e hidrólise do bagaço pré-tratado com combinado enzimático dos fungos Penicillium viridicatum RFC3 e Trichoderma reesei QM9414. 2011. 62f. Dissertação (Mestrado), Universidade Estadual Paulista Júlio de Mesquita Filho, Instituto de Biociências, Letras e Ciências Exatas, São José do Rio Preto, 2011. 